What Scientific Applications can Benefit from Hardware

Transactional Memory?

M. Schindewolf, B. Bihari, J. Gyllenhaal, M. Schulz, A. Wang, W. Karl

June 7, 2012 
This document was prepared as an account of work sponsored by an agency of the United States government. Neither the United States government nor Lawrence Livermore National Security, LLC, nor any of their employees makes any warranty, expressed or implied, or assumes any legal liability or responsibility for the accuracy, completeness, or usefulness of any information, apparatus, product, or process disclosed, or represents that its use would not infringe privately owned rights. Reference herein to any specific commercial product, process, or service by trade name, trademark, manufacturer, or otherwise does not necessarily constitute or imply its endorsement, recommendation, or favoring by the United States government or Lawrence Livermore National Security, LLC. The views and opinions of authors expressed herein do not necessarily state or reflect those of the United States government or Lawrence Livermore National Security, LLC, and shall not be used for advertising or product endorsement purposes.

This work performed under the auspices of the U.S. Department of Energy by Lawrence Livermore National Laboratory under Contract DE-AC52-07NA27344. 


\title{
What Scientific Applications can Benefit from Hardware Transactional Memory?
}

\author{
Martin Schindewolf*, Barna Bihari ${ }^{\dagger}$, John Gyllenhaal ${ }^{\dagger}$, Martin Schulz $^{\dagger}$, Amy Wang ${ }^{\ddagger}$, Wolfgang Karl* \\ ${ }^{*}$ Karlsruhe Institute of Technology, \{schindewolf,karl\}@kit.edu \\ $\dagger$ Lawrence Livermore National Laboratory, \{bihari1,gyllenhaal1,schulzm\}@1lnl.gov \\ ${ }^{\ddagger}$ IBM/Toronto, aktwang@ca.ibm.com
}

\begin{abstract}
Achieving efficient and correct synchronization of multiple threads is a difficult and error-prone task at small scale and, as we march towards extreme scale computing, will be even more challenging when the resulting application is supposed to utilize millions of cores efficiently. Transactional Memory (TM) is a promising technique to ease the burden on the programmer, but only recently has become available on commercial hardware in the new Blue Gene/Q system and hence the real benefit for realistic applications has not been studied, yet.

This paper presents the first performance results of TM embedded into OpenMP on a prototype system of BG/Q and characterizes code properties that will likely lead to benefits when augmented with TM primitives. We first, study the influence of thread count, environment variables and memory layout on TM performance and identify code properties that will yield performance gains with $T M$. Second, we evaluate the combination of OpenMP with multiple synchronization primitives on top of MPI to determine suitable task to thread ratios per node. Finally, we condense our findings into a set of best practices. These are applied to a Monte Carlo Benchmark and a Smoothed Particle Hydrodynamics method. In both cases an optimized TM version, executed with 64 threads on one node, outperforms a simple TM implementation. MCB with optimized TM yields a speedup of 27.45 over baseline.
\end{abstract}

\section{INTRODUCTION}

Achieving efficient and correct synchronization of multiple threads is a difficult and error-prone task. The correct use of lock based schemes requires a strict coding discipline to place matching lock and unlock operations into the code in a way that avoids race conditions and/or deadlocks. Additionally, lock-based synchronization often leads to high-overheads, either due to lock contention, when using coarse grained locks, or unnecessary lock overhead, when using fine grained locks. This not only slows down the overall process using the locks, but also has a global effect in large scale programming due to the creation of skew between processes as well as load imbalance, both major factors limiting the scalability of applications.

Transactional Memory (TM) has been proposed almost a decade ago to tackle these issues in shared memory systems [1]. TM simplifies synchronization by providing a single simple construct: the programmer wraps the critical instructions in a transaction (also called atomic block). These transactions are then executed optimistically in parallel and conflicting accesses are resolved by a TM run time system. As a consequence only the effects of entire and completed transactions are visible to concurrent threads, avoiding the visibility of intermediate memory states.

Except for a few, by now discontinued prototype implementations in research processors, TM has mainly been confined to software solutions and therefore has been burdened with significant runtime overheads. These overheads severely restrict its applicability with the consequence that non performance critical areas, for which the increase in programmability and ease of verification justify the additional cost, are the primary target. In high performance computing, however, the applicability of these approaches has been limited.

The recently introduced Blue Gene/Q (BG/Q) system by IBM for the first time provides Hardware Transactional Memory (HTM) in a commercially available platform. BG/Q is designed as a large scale platform for scientific computing workloads. The first full machine will be installed at Lawrence Livermore National Laboratory and will provide more than 1.6 million compute cores with a total of over 6 million hardware threads, making application scalability one of the premier challenges on this machine.

This paper presents the first performance evaluation of the HTM capabilities on BG/Q from the application perspective. Not every lock-based application will be able to benefit from HTM and it is important to understand what code properties lead to efficient executions and, hence, which codes can benefit from a port to HTM. In order to help code developers with this task, we provide a precise evaluation of the strengths and weaknesses of the architecture as well as what is required to map applications to the architecture in an efficient way. In particular, we focus on the synchronization primitives for parallel programming in shared memory architectures with OpenMP and provide detailed benchmark results. Our experiments take into account the application's characteristic (high or low contention), the influence of environment variables, the effects of enlarging transaction sizes, and hybrid parallelization with MPI. We apply our results to the optimization of a Monte Carlo Benchmark (MCB) that functions as a proxy application for several large scale Monte Carlo simulations. A Smoothed Particle Hydrodynamics method from the PARSEC benchmark suite serves as our second case study.

Specifically, we make the following contributions:

1) We introduce a new benchmark, CLOMP-TM, that is aimed at evaluating TM systems for scientific workloads. 
2) We characterize the performance of HTM combined with OpenMP on the Blue Gene/Q architecture using CLOMP-TM.

3) We study the influence of thread count, environment variables and memory layout on TM performance.

4) We evaluate MPI with OpenMP and multiple synchronization primitives to determine a fitting task to thread ratio for one node.

5) We identify code properties that are likely to yield performance gains with TM.

6) We condense the findings into best practices and apply them to a realistic Monte Carlo Benchmark code and a Smoothed Particle Hydrodynamics method.

For both case studies, an optimized TM version, executed with 64 threads on one node, significantly outperforms a simple or naive TM version validating the the best practices derived from our observations with CLOMP-TM.

The remainder of this paper is organized as follows. Section II provides background on Transactional Memory in general as well as related work. Section III describes our experimental setup, the TM architecture of the BG/Q system, and our benchmark used to determine overheads. Section IV presents low-level measurements, followed by the lessons learned in Section V. Section VI shows how we can use our lessons to add transactions to a Monte Carlo code and to a Smoothed Particle Hydrodynamics method. Section VII concludes and presents ideas for future work.

\section{BACKGROUND AND RELATED WORK}

Transactional Memory has been proposed as architectural support for lock-free data structures in shared memory systems [1]. The core idea is to replace pessimistic synchronization, such as locks, with optimistic synchronization in the form of transactions. Programmers can group updates into transactions and these can be executed concurrently with the rest of program. A runtime system (in hardware or software) detects conflicts between transactions as well as between a transaction and the rest of the program and, if necessary aborts and rolls the effects of the transaction back. As a consequence, the effects of any transaction are seen as if the transaction occurred as one atomic block, providing the necessary synchronization guarantees.

This concept does not only have performance implications, since contention free cases have the potential to execute concurrently and are not forced to be serialized as with pessimistic schemes, but it also, and perhaps more importantly, has the potential to provide a boost in programmability and maintainability. Lock based schemes are often error prone and require a strict coding discipline to ensure proper synchronization by placing matching lock and unlock instructions without causing deadlocks. The latter is particularly critical for fine grain locks. TM approaches, on the other hand, only expose a single concept, a transaction, to the user in the form of code blocks, which can be easily annotated within the source code. Many different TM designs have been proposed [2]. Software-based approaches [3], [4], [5], [6] (STM) use a
Software Transactional Memory library to implement algorithms for the detection and resolution of conflicting memory accesses. Software is very flexible but also comes with inherent overheads [7]. On the contrary Hardware Transactional Memory systems are fast for transactions that fit into the restricted hardware [8], [9], [10]. Further, hybrid approaches, combining hardware and software to accelerate execution and lift the limits of the hardware have been researched [11], [12], [13], [14].

The only paper that described an early experience with a commercial hardware transactional memory implementation published in a major conference, to our knowledge, is by Dice et al. [15]. The paper describes and evaluates the hardware transactional memory feature of SUN's Rock processor [16], which is no longer available. The focus of their paper is on the evaluation of concurrent data structures such as Red Black trees and Hashtable, and the construction of a minimum spanning forest [17]. The parallelization of these codes uses threads only. Thus, no experiments are made that estimate the performance of a hybrid parallelization with MPI. Further, there is an important difference between the HTM implementations of Rock and BG/Q. Rock is a checkpointbased architecture which is exploited in the context of TM to save and restore the architectural state of the registers in hardware. In BG/Q the TM runtime performs this task in software. This important difference will affect the performance of both architectures and makes transferring results of previous studies from the Rock to the BG/Q architecture extremely difficult.

A description of a second commercial HTM implementation can be found in a paper by Dick [18]. The goal is to accelerate the synchronized keyword in Java. Thus, no extensions to the language are made and explicit programming with transactions is not possible. Instead a heuristic decides whether to run a critical section as transaction or not.

Most STM papers use STAMP, a benchmark suite for transactional memory research [19]. The codes comprise: Bayesian network learning, gene sequencing, network intrusion detection, K-means clustering, maze routing, graph kernels, a client/server travel reservation system, and delaunay mesh refinement. This covers many application areas in which STMs have been used, but do not represent codes from the area of high performance computing, for which HTM is a promising approach to overcome synchronization overheads and to improve scaling of hybrid thread/MPI codes. In this paper, we therefore focus on a new benchmark explicitly designed to cover this area and present results that demonstrate how HTM can be deployed in HPC.

\section{EXPERIMENTAL SETUP}

For all following experiments we use an early prototype of BG/Q installed at IBM. TM is available as HTM through IBM's XL C/C++/Fortran Compiler suite for BGQ, which provides new language primitives that allow users to specify transactions. 


\begin{tabular}{l|l|l} 
Name & Description & Contention \\
\hline None & Threads do not conflict. & No contention. \\
Adjacent & Adjacent memory addresses are updated. & No to small contention. \\
Random & Randomly (but repeatable) seeming updates. & High contention. \\
FirstParts & Only the first parts are updated & Highest contention.
\end{tabular}

TABLE I

DIFFERENT CONTENTION LEVELS IN THE CLOMP-TM BENCHMARK.

\begin{tabular}{l|l|l} 
Name & Implementation & Description \\
\hline Bestcase & - & Bestcase without synchronization. \\
Serial Ref & - & Serial reference implementation. \\
\hline Small TM & \#pragma tm_atomic & Synchronizing each update with a transaction. \\
Small Atomic & \#pragma omp atomic & Synchronizing each update with an atomic operation. \\
Small Critical & \#pragma omp critical & Synchronizing each update with OpenMP's critical section. \\
\hline Large TM & \#pragma tm_atomic & All scatter zone updates in one transaction. \\
Large Critical & \#pragma omp critical & All scatter zone updates in one critical section. \\
\hline Huge TM X & \#pragma tm_atomic & X times Large TM in one transaction. \\
& & TABLE II
\end{tabular}

\section{A. Overview of BG/Q's TM Hardware}

The BG/Q prototype we had access to contained 32 nodes with 16 cores each. Each core can execute up to four hardware threads. Transactional memory is implemented within the L2 cache, which consists of 16 banks of $2 \mathrm{MB}$ each located across a full crossbar from the 16 multithreaded compute cores. The L2 cache has a cache line size of 128 Bytes. Memory accesses that can lead to conflicts between transactions, are tracked by the L2 cache, which is a point of coherency. Conflict detection between different transactions is completed in hardware, while conflict resolution is coordinated through the TM software stack. Note that, in addition to TM, the L2 cache also implements an improved set of atomic operations that also target faster and more efficient thread synchronization. Comparisons in the remainder of the paper between TM and atomic operations therefore provide results between two novel and highly optimized schemes. More information on BG/Q's hardware in general can be found in a recent presentation by Haring at Hot-Chips [20].

\section{B. Application Perspective in BG/Q's TM Software Stack}

By default, the TM runtime defaults to a 'lazy' (or optimistic) conflict detection scheme, at commit time, as the runtime suppresses the hardware from sending conflict interrupts to the conflicted threads. However, applications/users can enable a 'pessimistic detection' scheme by setting the environment variable TM_ENABLE_INTERRUPT_ON_CONELICT. That is, conflict arbitration happens immediately at the time of conflicts. Either scheme needs to be carefully chosen as an already doomed thread, if allowed to run till the end, may cause further spurious conflicts.

The TM runtime also relies on a lazy versioning (i.e., write-back) scheme as all speculative writes are buffered in the multi-versioned cache until commit time. Strong atomicity (i.e., opacity) is guaranteed unless a thread is running in irrevocable mode. In such a case, the thread runs non-speculatively and all writes take affect immediately.
The TM_MAX_NUM_ROLLBACK environment variable controls when a thread should enter into irrevocable mode. The irrevocable mode is a mechanism that guarantees that a thread makes progress. The contention manager favors an older thread to commit based upon the timebase register value of the thread at the time when speculation starts. Aborting a transaction does not back-off for pre-determined time, rather, a thread retries immediately. The runtime also implements flat nesting whereby commits and rollbacks are to the outermost TM region. As an additional feature, the runtime monitors the TM behavior of the application and provides the resulting TM statistics to the user. All TM statistics, presented in this paper, are retrieved by this method.

\section{The CLOMP-TM Benchmark}

Since current TM benchmark suites do not provide the necessary coverage for scientific applications, we focus on the development of a new benchmark specifically designed to expose the range of properties needed to characterize scientific workloads, CLOMP-TM ${ }^{1}$. It is designed to compare multiple synchronization constructs and approaches over a workload that is typical for scientific codes. In fact, it was created to help us mimic the application characteristics of several large scale, multi-physics applications used in production at DOE laboratories. It achieves this flexibility and wide coverage through a series of knobs that allow us to explore varying transaction granularities and conflict rates, coupled with typical computational kernels found in scientific codes.

CLOMP-TM originates from the publicly available CLOMP benchmark [21] used for evaluating OpenMP implementations. CLOMP resembles an unstructured mesh with a set of partitions. Each partition holds a linked list of zones. To vary the pressure on the memory system, the size of these zones can be configured. Computation, that is performed when updating

\footnotetext{
${ }^{1}$ For the experiments CLOMP-TM version 1.59 is used and will be made publicly available at publication time of the paper.
} 


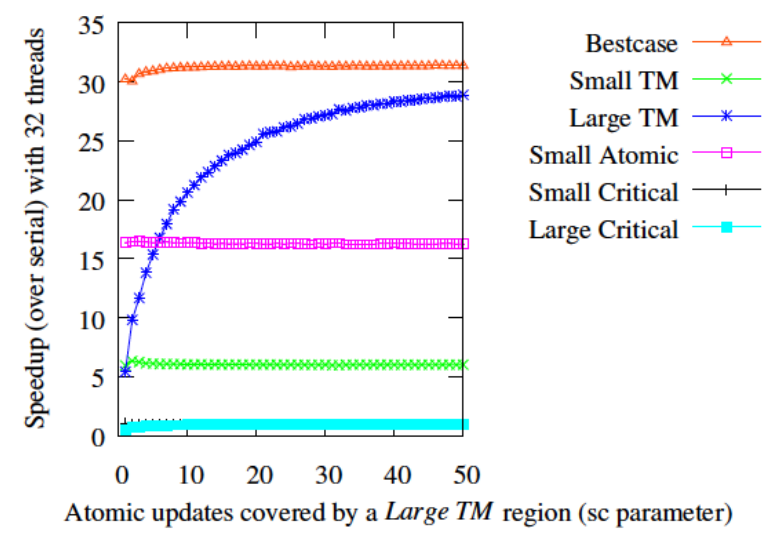

(a) 32 threads.

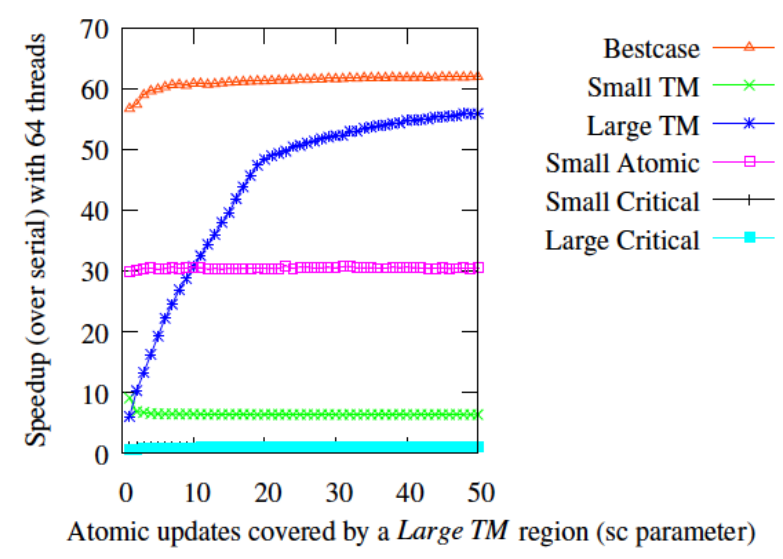

(b) 64 threads.

Fig. 1. CLOMP-TM performing 8 divide operations with a stride of 4 per zone update with excellent speedups of Large TM over Small Atomic. Run with clomp-tm-bgq-divide4 -1 1256128256 stride1,1, stride1\%/2 sc 1006100.

a zone, only uses the first 32 bytes of each zone. The computation per zone update can be scaled by a factor. While the original CLOMP aims to quantify overheads due to threading and the specific OpenMP implementation, the CLOMP-TM aims at quantifying and comparing synchronization overheads of multiple synchronization constructs. CLOMP-TM can be configured to resemble the synchronization characteristics of typical scientific applications used in HPC. Thus, performance results of CLOMP-TM not only enable us to provide detailed characteristics of the low-level properties of the Blue Gene/Q hardware, but also, and more importantly, to project the performance impact of TM on large scale parallel applications.

Major changes over CLOMP: In order to study the impact of TM in the presence of loop dependencies and the resulting conflicts, CLOMP-TM adds explicit and controllable dependencies to the loop structures of CLOMP. Besides the implementation with TM, CLOMP-TM also tests optimistic execution not secured by any synchronization ${ }^{2}$ or using other constructs such as atomics or OpenMP-based constructs with the same level of abstraction in terms of programming.

For a meaningful comparison of optimistic and pessimistic synchronization constructs, multiple memory access patterns have to be considered. These memory access patterns determine the likelihood of a conflict between concurrent accesses of two threads. A single parameter defines the zones that are updated by a thread. The contention arises when multiple threads update the same zones. These different contention scenarios are shown in Table I.

In comparison to the CLOMP benchmark, the updates of a zone are enlarged. This new construct is called "scatter zone" and enables larger critical sections, which resembles the update of multiple variables (e.g., coordinates with multiple dimensions $x, y$, and $z$ ) in one critical section. For the large versions of the synchronization constructs, scatterCount

\footnotetext{
${ }^{2}$ In this configuration CLOMP-TM does not return correct results, but the timings can be used to study conflict free cases.
}

updates many zone in a single synchronized block.

Each iteration executes the selected computation pattern. Available patterns with increasing complexity are: none, divide, manydivide, and complex. CLOMP-TM is carefully designed to eliminate as much noise as possible: $\mathrm{I} / \mathrm{O}$ is performed only outside of timing loops and all the loops are run just before the timing loops to eliminate start up costs and cold cache effects. Table II holds the synchronization constructs to be compared.

Comparison of CLOMP-TM with TM benchmarks: Apart from the heavily cited STAMP benchmark suite [19] that does not represent the scientific application behavior we are interested in, a growing number of parameterized workloads gains popularity. An important example is the WormBench workload [22]. WormBench is derived from the popular snake game and has been designed to evaluate and verify the efficiency of a TM system. WormBench is written in $\mathrm{C \#}$ and enables to compare TM performance with a global lock. Parameters are size of the world (matrix), number of worms (threads), body and head size of a worm and operations to be performed while moving. In contrast CLOMP-TM enables a comparison with a single instruction atomic update, an unsynchronized version and two sizes of transactions and critical sections respectively. For our workloads C\# does not play an important role and we believe that a more versatile benchmark to model scientific workloads is needed and thus propose CLOMP-TM. A more suited candidate for the modeling of arbitrary TM workloads is Eigenbench [23]. Eigenbench uses orthogonal metrics to model a specific workload. For our use case, not knowing a priori how TM will perform, we would have to transactify the application, measure the metrics, derive a configuration for Eigenbench and use this to model our workload. With CLOMP-TM, the user only needs to know the number of shared memory accesses and the number of floating point operations per loop iteration of an application to set the right parameters that will resemble the application behavior. 


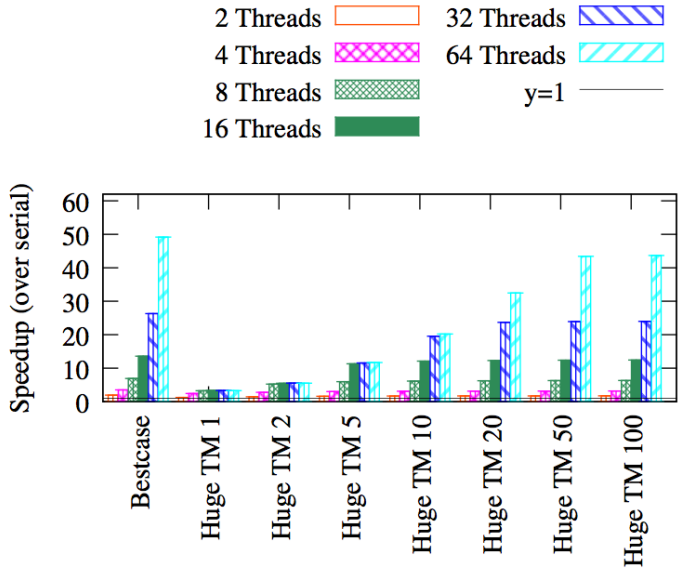

Fig. 4. CLOMP-TM performing 8 divide operations per zone update with huge critical sections. Speedup shown with None - generated with CLOMPTM version 1.36

\section{Characterizing TM Performance On BG/Q USING CLOMP-TM}

For the CLOMP-TM cases presented in this paper, synchronization overheads can dramatically affect speedup. We vary the parameters of CLOMP-TM to learn how the parameters affect the speedup and to find out what code properties qualify for TM. These results will help application developers to tune their codes (e.g., through picking a better suited synchronization primitive), but the achievable speedup is determined by the properties of the application (e.g., ratio of computation and synchronization, contention for memory locations).

For our initial experiments targeted at understanding the potential for TM, we chose the parameters for CLOMP-TM in a way that TM outperforms a highly efficient implementation of omp atomic. In this configuration, CLOMP-TM performs 8 divide operations per zone update with a stride of 4 and the results are shown in Figure 1. Threads do not contend for memory locations. We increase the size of the scatter zone so that an increasing amount of updates is carried out in Large TM. Figure 1 a) illustrates that in the case of 32 threads performing 4 zone updates is the cross-over point for Large TM over Small Atomic. For 64 threads the number of zones is twice as high (see Figure $1 \mathrm{~b}$ )). Please note that the large amount of computation per zone update masks the overheads of synchronization.

\section{A. Synchronization Overhead}

To understand the tradeoffs in using TM, we first study the synchronization overhead associated with different approaches and contrast them to the TM results. We obtain the results in this section by using the parameters shown in Table III. Memory is allocated by the main thread. This is sufficient because memory access are uniform in BG/Q. The setting of zonesPerPart equal to 100 stems from the original CLOMP and mimics the loop sizes of many multiphysics applications [21]. The chosen computation pattern is divide. For each zone update 8 extra divide calculations are executed. The environment variable OMP_WAIT_POLICY has been set to ACTIVE for all runs.

$$
\begin{array}{r|l}
\text { numParts } & 64 \\
\text { zonesPerPart } & 100 \\
\text { zoneSize } & 128 \\
\text { zone alignment } & 128 \\
\text { scatter } & 3 \\
\text { flopScale } & 1 \\
\text { timeScale } & 100 \\
\text { Zones per Part } & 100 \\
\text { Total Zones } & 6400 \\
\text { Zone Calc Stride } & 1 \\
\text { Extra Zone Calcs } & 8 \\
\text { Zone Calc Flag } & - \text { DDIVIDE_CALC } \\
\text { Zone Calc Formula } & ((1.0 /(x+2.0))-0.5)
\end{array}
$$

TABLE III

PARAMETERS FOR CLOMP-TM.

Figure 2 shows the speedup of the different synchronization mechanisms for updating one memory location. In case of high contention, generated by the Random and firstParts memory access pattern, Small Atomic is the method of choice for synchronization.

Large TM outperforms Large Critical as can be seen for both no and high contention cases of Figure 3. Thus, for critical sections with more than one memory update, TM is the preferred method. The Huge TM with 100 times the size of Large TM performs excellent in case of no contention (cf. Figure 4). Further experiments with higher contention cases reveal that this speedup is very fragile. These experiments demonstrate (and the TM statistics confirm) that longer transactions are more susceptible to contention.

\section{B. Conflict Probability}

The performance of any TM application depends on the number of conflicts it has to encounter that lead to potentially costly rollbacks. We study this issue by extending CLOMPTM with a special mode that allows to transition between scatter modes. Thus, a parameter has been added that defines the number of intended conflicts for this run. For our experiments this parameter can be computed from a conflict probability ( $c p$ ) according to the following equation: total zones $*$ scatter $* c p$. Updates are counted as intended conflicts and performed inside a large transaction. Note, however, not all of these intended conflicts lead to an actual conflict and some conflicts can cause multiple rollbacks.

Figure 5 a) illustrates the impact of the number of retries on the achievable speedup. From this figure we can clearly see that a linear increase of the conflict probability (shown as intended conflicts) leads to an exponential decrease of the speedup. In this experiment the zone size is set to 128 bytes. In case it is smaller (e.g., 64 or 32 bytes) conflicts may be falsely detected because two zones are mapped to the same cache line. These False Positives are eliminated when the zone size equals the size of the L2 cache line. 


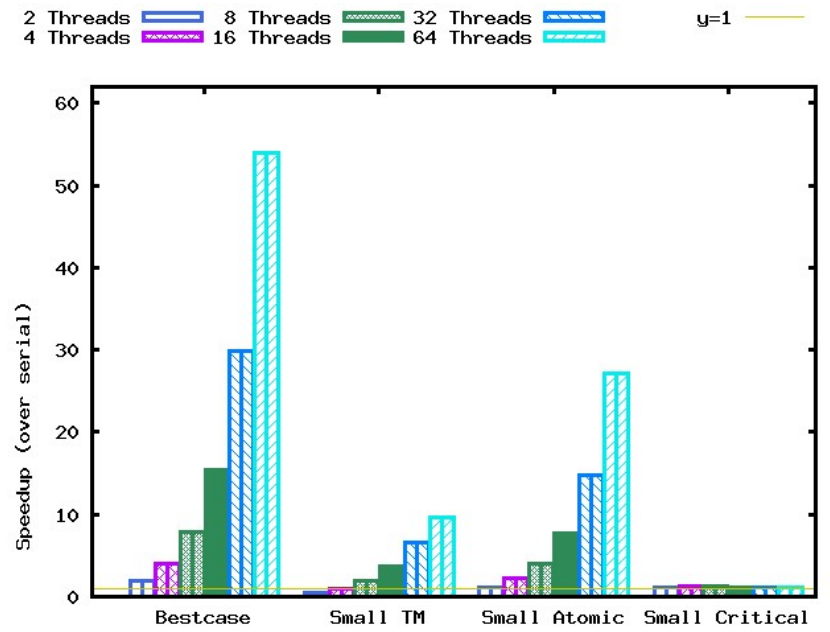

(a) Speedup with None.

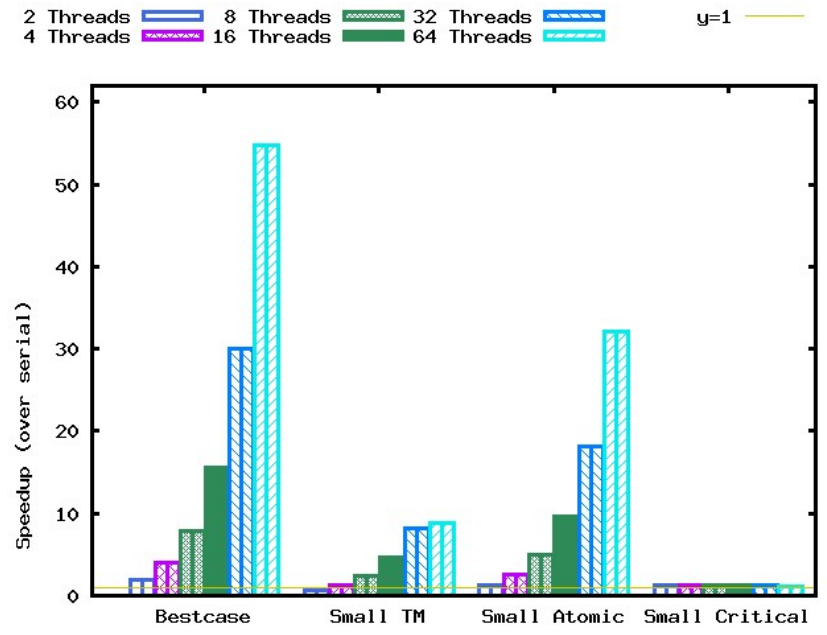

(b) Speedup with Random.

Fig. 2. CLOMP-TM performing 8 divide operations per zone update with small critical sections.

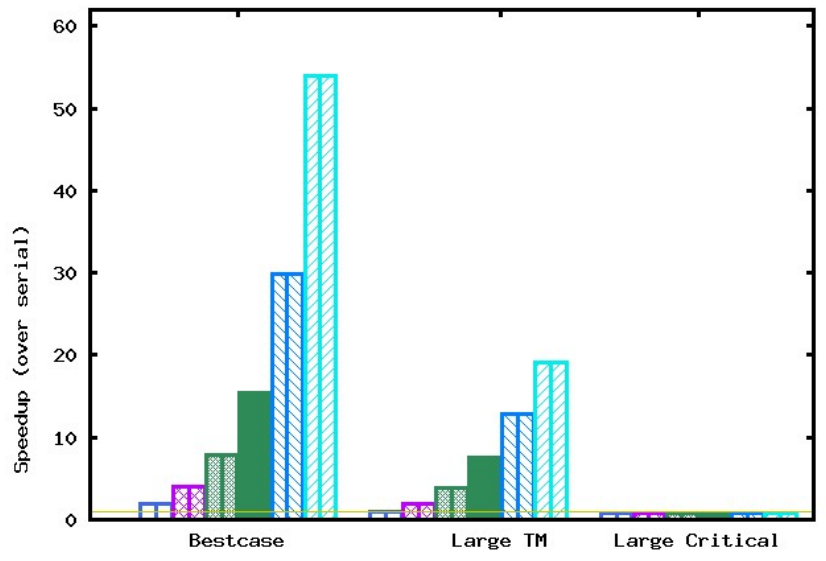

(a) Speedup with None.

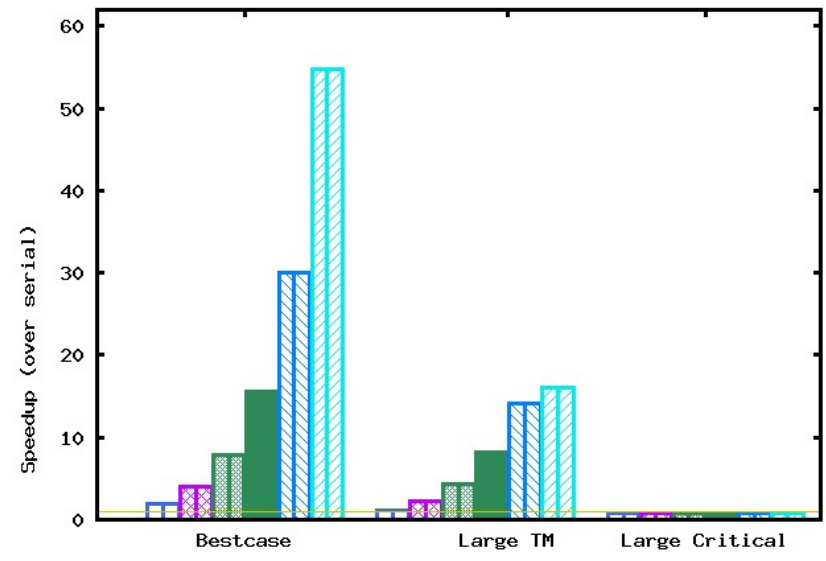

(b) Speedup with Random.

Fig. 3. CLOMP-TM performing 8 divide operations per zone update with large critical sections.

\section{Tuning the BG/Q TM Runtime Environment}

The TM runtime system in BG/Q provides a series of knobs that can be used to fine tune the performance of HTM applications. These knobs are available to the user through environment variables that can be set before the code's execution. The most significant one is TM_MAX_NUM_ROLLBACK (RBM), which controls the number of times a transaction can be aborted and rolled back before the runtime gives up on it and executes it in irrevocable mode, i.e., the transaction is executed non-transactional under a global lock so that other transactions can not interfere. The TM runtime will further mark this transaction and execute it in irrevocable mode right away on a subsequent execution.

Figure 5 shows results with 32 threads and RBM set to 1 and 10. In Figure 5 a) RBM is set to 10 and shows a significant higher number of retries than Figure 5 b) (RBM 1). The relative number of serialized transactions is higher for $\mathrm{RBM}=1$. Both observations are due to the RBM setting because a smaller RBM value serializes after less retries. In terms of speedup RBM 10 outperforms RBM 1 because of the less frequent serialization. The effects of the adaptive TM runtime on the performance need a closer investigation.

Figure 6 demonstrates the influence of RBM1, RBM 5 and RBM 10 on the achievable speedup with TM. For all contention levels and Small TM the differences between RBM 5 and RBM 10 are insignificant. For higher contention and Large TM, RBM 10 has slight advantages over RBM 5. RBM 1 shows the worst performance for the presented level of contention.

An second important parameter for TM is the scrub rate. It triggers a garbage collection for TM SpecIds. SpecIds mark entries in the cache as belonging to the same or different transactions. Figure 7 shows that varying the scrub rate has a large impact. For our benchmark with a lot of transactions and short intervals between these, a scrub rate of 6 shows the best performance. 


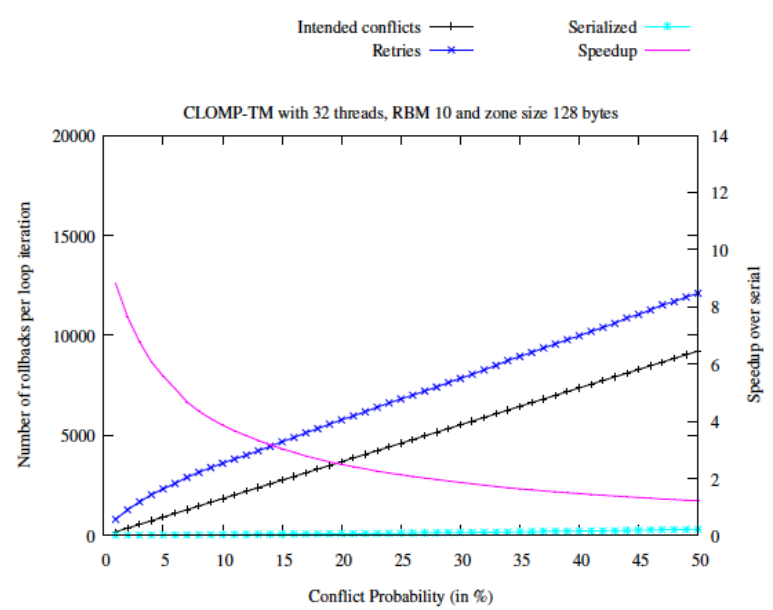

(a) 32 threads with RBM 10 .

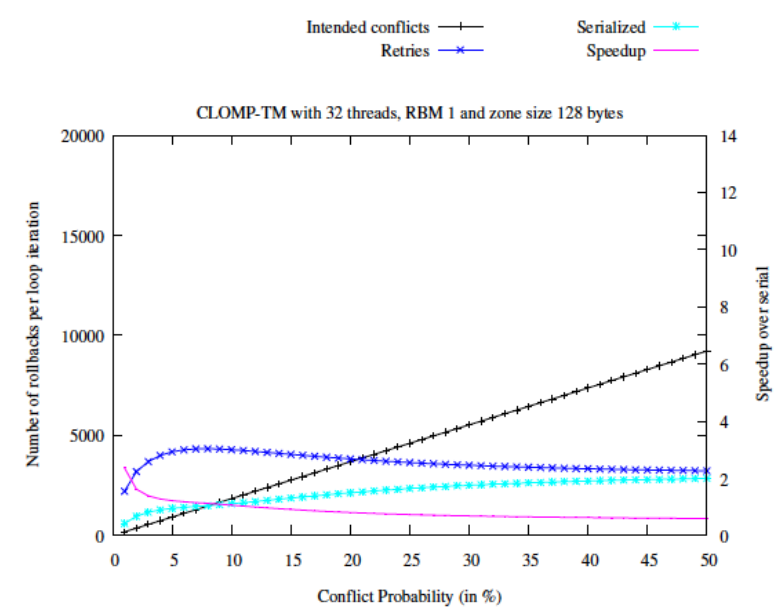

(b) 32 threads with RBM 1.

Fig. 5. Influence of TM_MAX_NUM_ROLLBACK (RBM) on retries/speedup. Run with clomp-tm-bgq-divide1 -1 1 x 1 d6144 128 firstzone, $c p$, randFirstzone 31006100.

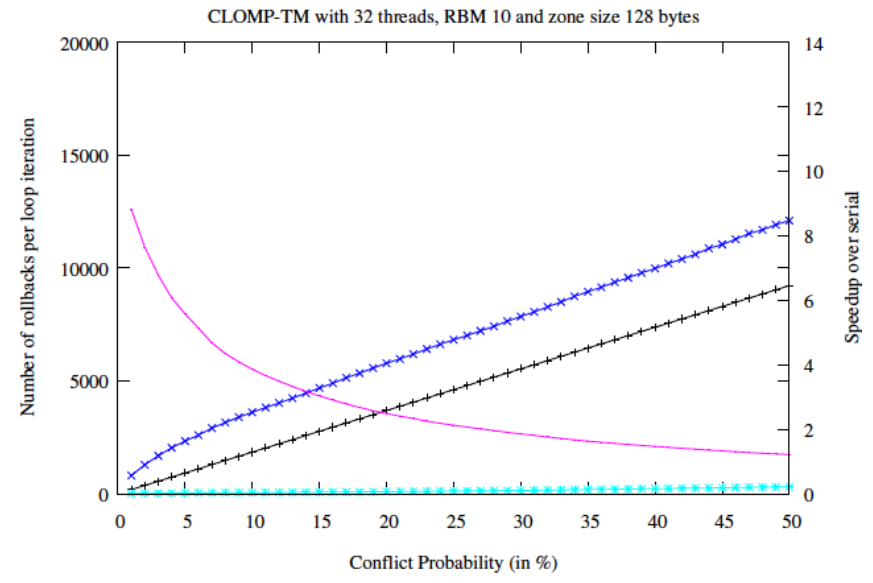

(a) 32 threads with RBM 10 .

Fig. 6. Studying the influence of setting TM MAX NUM ROLLBACK with CLOMP-TM and changing the level of contention. Run with clomp-tm-bgq-divide4 321256128256 stride1, $c p$, stride1\%/2 101006100.

\section{CLOMP-TM with mixed Scatter Modes}

So far, we have only discussed settings with a single scatter mode at a time (cf. Table I). This leads to a fixed TM application behavior that defines the contention between threads for the whole program run. As a result, TM either performs excellent because of the lack of conflicts (e.g., scatter mode None) or suffers from the frequent retries (e.g., firstParts). This model, while useful to get point results, is too restricted to model all scientific workloads and expose the potential of TM. CLOMP-TM therefore additionally supports an two different scatter modes that execute concurrently. It uses a parameter to define how often the second scatter mode will be used for updates. Increasing this parameter leads to more updates with the second scatter mode.
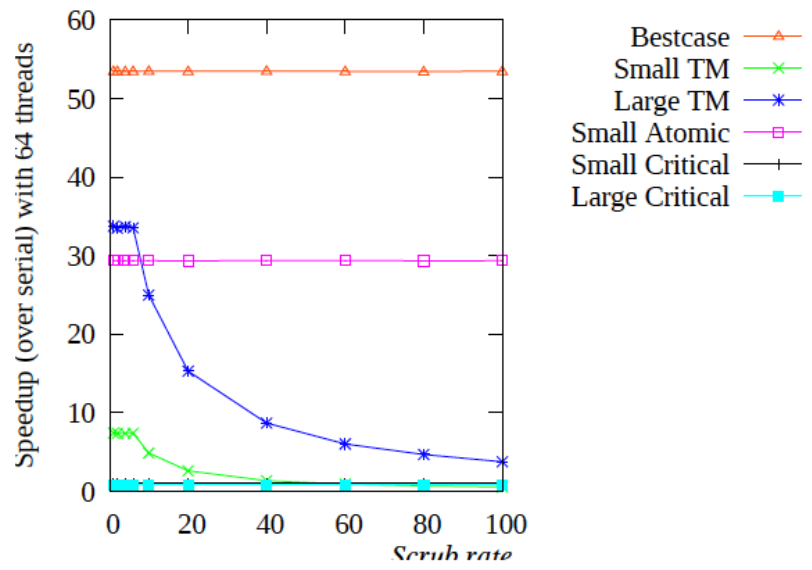

Fig. 7. Influence of the scrub rate for SpecIds on performance with 64 threads. Run with clomp-tm-bgq-divide1 -1 164100128 InPart, 10, firstParts 1010 sr 100

\section{E. Using TM in the Context of MPI Applications}

Up to this point, we focused on single node experiments using OpenMP as the method for threading. To work across nodes and hence to exploit the vast parallelism available in the planned BG/Q system, scientific applications will require additional parallelization with MPI in order to exploit its compute power. Consequently, it is important to understand the interplay between OpenMP threading with TM support and having multiple MPI tasks on the node.

In the following we study the side effects of running multiple MPI tasks, each executing CLOMP-TM, on one node. Our goals are:

1) to verify the robustness of the previously presented results,

2) identify bottlenecks due to the sharing of architectural resources, 

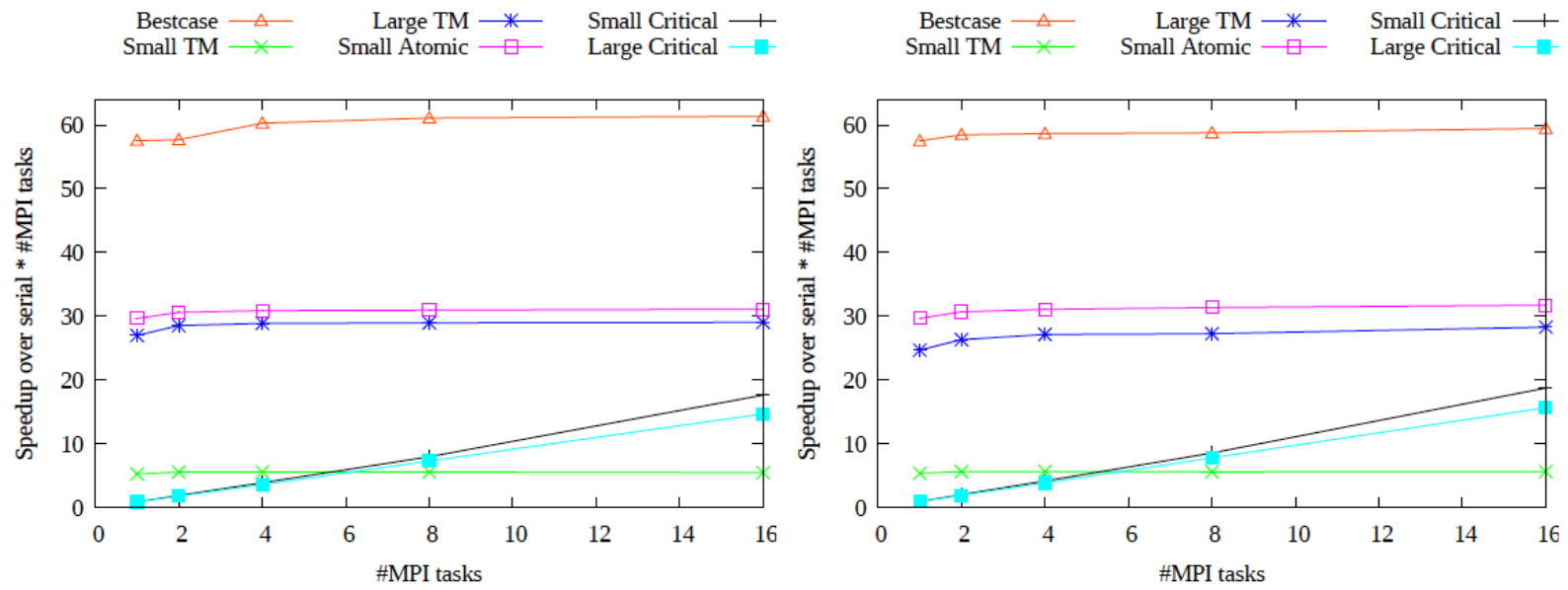

Fig. 8. CLOMP-TM with MPI performing 8 divide operations with a stride of 4 per zone update with no and high contention. Run with

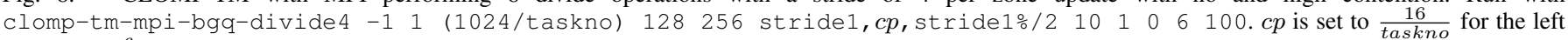
and $\frac{1.12 * 10^{6}}{\operatorname{taskno}}$ for the figure on the right.

3) and determine a fitting MPI task to OpenMP thread ratio.

Similar to the previously published CLOMPI [21], we designed CLOMP-TM to execute multiple instances of its core functionality, synchronized by MPI operations. Besides calls to init and finalize MPI, we inserted MPI_Barriers. These barriers are placed such that all MPI tasks execute the code for the same synchronization primitive. An example for the placement of the MPI_Barrier calls is shown in Listing 1. To execute in this lock step fashion guarantees that all MPI tasks execute the code for the same synchronization primitive. As a consequence, we can directly control the contention on the architectural resources that are necessary for synchronization (such as the L2 cache). Thus, the methodology proivides a clear and controllable mechanism to study the architectural resources needed by individual synchronization primitives.

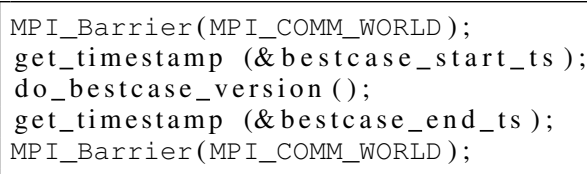

Listing 1. Use of MPI barriers for CLOMP-TM with MPI.

Figure 8 illustrates the performance characteristics of CLOMP-TM with MPI and small as well as large critical sections. The experiments are carried out as strong scaling experiments, i.e., the amount of work is constant for all task counts. We achieve this by dividing the number of parts (initially 1024) by the number of MPI tasks. The number of updates in the second scatter mode is also divided by the number of MPI tasks. All MPI tasks execute as many threads as possible without oversubscribing the node (e.g., 1 MPI task executes 64 threads).

First, Figure IV-E a) shows the average speedup of the threads in each MPI task multiplied by the number of MPI tasks on the y-axis. The number of MPI tasks is plotted on the $\mathrm{x}$-axis. In this case with extremely low contention, Large TM performs almost as well as Small Atomic. The surprise is that for large task counts, Small and Large Critical perform better than Small TM. Especially Small Critical, that is protecting one memory location, has been optimized heavily in the new BG/Q L2 cache and is now a strong alternative for TM if the granularity in the codes allows for this. With increased contention, as shown in Figure IV-E, Large TM and Small TM perform a little worse than before but the overall trend with respect to the critical section is still the same. Large Critical benefits from the smaller thread numbers at higher task counts because the cost for serialization is reduced.

\section{F. Finding a Competitive Task to Thread Ratio}

The architecture of one BG/Q node features 16 compute cores each equipped with 4-way hyper-threading. As demonstrated in earlier papers [21], an OpenMP barrier has a higher overhead for higher thread counts. Thus, a hybrid parallelization with MPI and OpenMP may achieve higher performance than an OpenMP only implementation. In order to be able to compare results of OpenMP and hybrid parallelization, we use a simple metric. For the hybrid case, we multiply the reported OpenMP speedup with the number of MPI tasks. Figure 8 shows that the Bestcase across MPI tasks is stable. Depending on the properties of the application the best synchronization primitive varies. Across all tested memory access patterns and MPI tasks configurations, the OpenMP version with the highest possible thread count performs best. While this is not surprising since the BG/Q architectures requires at least two threads per core to be able to reach full issue bandwidth, it is nevertheless an important first insight that we gain from this experiment. For architectures with hyper-threading, the additional HW threads are often turned off because they lead to a slowdown. For the BG/Q architecture running the CLOMP-TM (with MPI) benchmark this is not the case. Every thread (even beyond the minimum of two needed for full issue bandwidth) contributes an important part of the reported performance. For the executed strong scaling experiments, 
however, the results of finding a preferable task to thread ration are inconclusive. All tested ratio perform well and differences are extremely small.

The synchronization primitive with the best performance varies. In all high contention cases Small Atomic performs best. For cases with little to no contention Large TM may perform almost as good as Small Atomic. The large transactions benefit from the optimistic concurrency and the overhead for setting up the transaction is amortized due to the long transaction size. Unfortunately, this effect is limited to scenarios where expensive roll back operations are infrequent.

\section{LESSONS LEARNED}

The experiments described above gives us a clear characterization of HTM on BG/Q and provide the necessary information to understand which kind of applications can benefit from HTW. In the following we summarize these findings in set of best practice guidelines that will help code developers on BG/Q decide if and how to best exploit HTM.

The identified preferable code properties for TM are:

- critical section should have low contention so that conflicts are unlikely,

- critical sections should access more than one memory location (preferably in the range of 10 to 20) so that omp atomic is not applicable and TM's property of providing atomicity for updates of multiple memory locations is valuable,

- high computation to synchronization ratio so that computation can mask the overheads of synchronization.

For synchronization with OpenMP, both the size of the code region that needs to be executed atomically and the potential conflict rate play an important role:

- For code regions that only require atomic updates using one instruction, omp atomic shows the best performance, since it can be mapped to the efficient atomic instructions implemented in the BG/Q L2 cache.

- For larger critical sections with low to moderate contention and conflict potential $(<<1$ rollback per transaction), TM using the $t m$ atomic primitive is beneficial, since the costs of conflicting transactions are amortized by avoiding serialization.

- In case of very high contention ( $>1$ rollback per transaction) and small critical sections, omp critical also outperforms TM, since TM conflicts and rollbacks start dominating leading to higher overhead.

- For applications that are not utilizing the full memory bandwidth with a high transactional execution time and short times in between transactions, setting the scrub rate to 6 yields better performance.

These findings complement a previous study on using Software Transactional Memory for scientific codes using a different and more specific setup [24]. Additionally, researchers already identified codes that match the criteria from above and are expected to benefit from TM [25], although also this work was limited to STM methods and has up to now not been verified on a HTM system, and thus no performance results have been published either. Our current recommendations verify the applicability of these previous preliminary studies to HTM, extend them by adding tradeoffs offered by the new performance knobs found in IBM's HTM solution, and generalize them to a more comprehensive guide for application developers.

\section{Application Case Studies}

\section{A. MCB: A Proxy Application for Monte Carlo Simulations}

In this section we apply the best practices from the previous section to a benchmark closely representing a real world application. The Monte Carlo Benchmark (MCB) models a Monte Carlo simulation, a popular technique for physics simulations. In contrast to classical simulation approaches, Monte Carlo simulations do not compute their result explicitly, but instead adaptively sample the simulation domain and execute individual simulations for each sample. This process is repeated until the probability of a result can be quantified.

The initial MCB code was already parallelized with MPI and OpenMP. The original version uses omp critical and omp atomic to synchronize OpenMP threads (denoted as Critical \& Atomic in the following). As a first, naive TM implementation, referred to as $T M$ naive, we replace all critical sections with transactions and set the TM environment variables to their default for TM simple and Critical \& Atomic.

Additionally, we create an optimized version, called TM opt, following the lessons in the previous section. In $T M$ $o p t$ we use a hybrid strategy matching the characteristics for each synchronization construct: synchronizations that involve only one instruction use omp atomic, while all omp critical constructs are replaced with tm_atomic.

Table VI-A shows the results of the experiments with one MPI task and 64 threads in a strong scaling experiment with $5 * 10^{6}$ particles. Each value is an average of samples per second over 30 runs and normalized to baseline: samples per second with one MPI task and one thread. TM opt performs very well with a speedup over baseline with 27.45 , which is slightly better than the original version but comes with reduced code complexity and programmer effort. The result of TM naive demonstrates that the lessons learned in this paper are essential to getting good performance. Further experiments reveal a limited potential for optimizing the synchronization of threads in MCB. Commenting out all occurrences of omp atomic and omp critical (and ignoring the fact that this results in wrong answers for the simulation) yields $\approx 5 \%$ perfomance improvement.

\begin{tabular}{l|r|r|r} 
Code version & Critical \& Atomic & TM naive & TM opt \\
\hline Speedup & 27.57 & 20.06 & 27.45
\end{tabular}

TABLE IV

MCB WITH ONE MPI TASKS AND 64 THREADS (STRONGSCALING) SPEEDUP OVER BASELINE. 


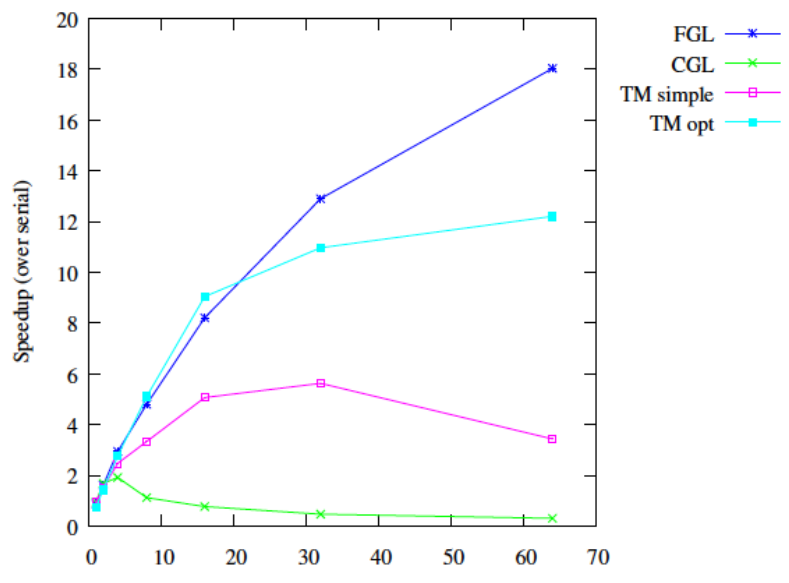

(a) Input data set simlarge.

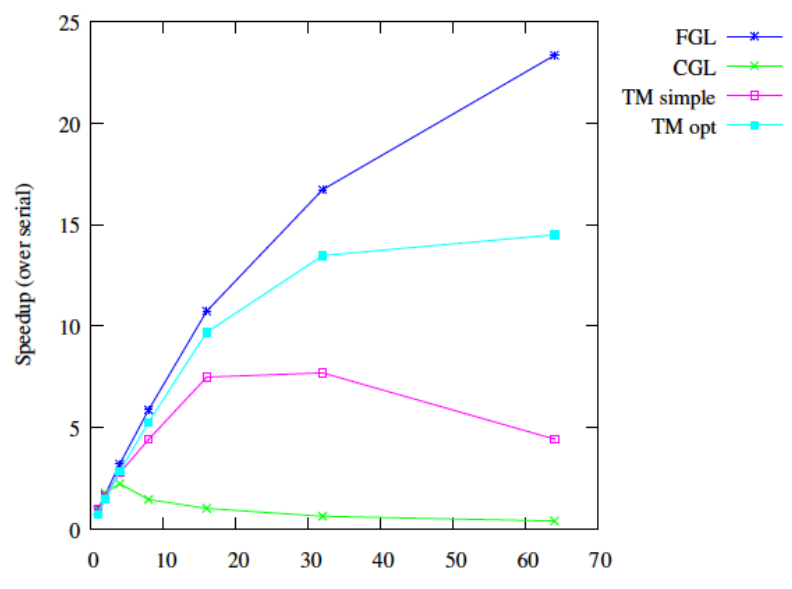

(b) Input data set native.

Fig. 9. fluidanimate with coarse grain and fine grain locks as well as simple and optimized transactions.

\section{B. Fluidanimate from the PARSEC suite}

In addition to the Monte Carlo Benchmark, we use the application fluidanimate from the PARSEC benchmark suite [26]. fluidanimate implements a Smoothed Particle Hydrodynamics (SPH) method to animate fluid dynamics. To include it in the PARSEC suite, the application has been parallelized with Pthreads and fine grain locking. Under the assumption that particles can not travel more than one cell in one time step, this parallelization uses an array of locks to protect the boundaries. An if-statement checks whether a lock needs to be taken. This synchronization pattern is rather sophisticated and exceeds the complexity of a single global lock by far. Because the programming complexity of TM can be compared with a single global lock, we added two versions: one with a coarse grain lock (cgl) and a simple TM version (TM simple) that replaced all lock acquire and lock release operations (that occur in three code segments) with a transaction.

Then, we apply the lessons learned from Section V. First, we enlarge all three transactions by removing the if-statement and changing the two outer loops to be inner loops. More measurements reveal that for the third transaction having three nested inner loops is best. Further, we reduce the scrub rate to 6 so that SpecIds from the TM hardware will be reclaimed faster. All measures combined deliver the performance shown for TM opt in Figure 9. For the small input data set (simlarge) and medium thread counts, TM opt outperforms the fine grain locking (cf. Figure 9 a)). For the larger input data set (native) the lessons learned are necessary to increase the scalability with TM and come into sight of fine grain locking (cf. Figure 9 b)). The execution with native input data sets increases the input data size and the frame rate simultaneously. We were interested to know which of these parameters influences the performance in favor of TM. The result is that the smaller input data favors TM whereas the frame rate simply serves as a multiplier of the observed performance. Further, this experiment reveals that the BG/Q architecture is extremely stable with very little noise. The observed performance of the synchronization patterns shows that even with Hardware Transactional Memory, expert-level use of lightweight efficient fine-grained locks will be hard to beat. Moreover, we identified the need to research tools with support for TM that enable an in-depth understanding of the TM behavior and the causes for performance degradation. From a programmability perspective, employing TM is as simple as using a single global lock. For this experiment, even the unoptimized TM version outperforms the single global lock in terms of speedup and scalability. Therefore TM takes an important step towards simplifying shared memory programming.

\section{CONCLUSIONS}

In this paper we evaluated BG/Q's TM hardware from the perspective of an application developer. We introduced, CLOMP-TM, a benchmark designed to represent scientific applications, and applied it to benchmark transactions against traditional synchronization primitives, such as omp atomic and omp critical. We then extended CLOMP-TM with MPI to mimic hybrid parallelization with OpenMP and MPI. Additionally, we studied the impact of environment variables on the performance. Finally, we condensed the findings into a set of best practices and applied them to a Monte Carlo Benchmark that closely resembles real world applications. An optimized TM version of MCB with 64 threads achieved a speedup of 27.45 over the baseline. Further, an optimized TM version of the Smoothed Particle Hydrodynamics method from the PARSEC suite significantly outperformed a simple TM version as well as a coarse grain lock and verified the usefulness of the best practices.

\section{ACKNOWLEDGMENT}

Part of this work was performed under the auspices of the U.S. Department of Energy by Lawrence Livermore National Laboratory under Contract DE-AC52-07NA27344. 


\section{REFERENCES}

[1] M. Herlihy and J. E. B. Moss, "Transactional memory: architectural support for lock-free data structures," in Proceedings of the 20th annual international symposium on computer architecture, ser. ISCA '93. New York, NY, USA: ACM, 1993, pp. 289-300. [Online]. Available: http://doi.acm.org/10.1145/165123.165164

[2] T. Harris, J. Larus, and R. Rajwar, Transactional Memory. Morgan \& Claypool Publishers, 2010, vol. 5, 2nd edition, Synthesis Lectures on Computer Architecture.

[3] P. Felber, C. Fetzer, and T. Riegel, "Dynamic performance tuning of word-based software transactional memory," in Proceedings of the 13th ACM SIGPLAN Symposium on Principles and practice of parallel programming, ser. PPoPP '08. New York, NY, USA: ACM, 2008, pp. 237-246. [Online]. Available: http://doi.acm.org/10.1145/1345206. 1345241

[4] D. Dice, O. Shalev, and N. Shavit, "Transactional locking ii," in Proc. of the 20th International Symposium on Distributed Computing (DISC 2006), 2006, pp. 194-208.

[5] Y. Lev, V. Luchangco, V. Marathe, M. Moir, D. Nussbaum, and M. O1szewski, "Anatomy of a scalable software transactional memory," in TRANSACT'09: Workshop on Transactional Computing, February 2009.

[6] B. Saha, A.-R. Adl-Tabatabai, R. L. Hudson, C. C. Minh, and B. Hertzberg, "Mcrt-stm: a high performance software transactional memory system for a multi-core runtime," in PPoPP '06: Proceedings of the eleventh ACM SIGPLAN symposium on Principles and practice of parallel programming. New York, NY, USA: ACM, 2006, pp. 187-197.

[7] C. Cascaval, C. Blundell, M. Michael, H. W. Cain, P. Wu, S. Chiras, and S. Chatterjee, "Software transactional memory: Why is it only a research toy?" Queue, vol. 6, no. 5, pp. 46-58, 2008.

[8] N. Njoroge, J. Casper, S. Wee, Y. Teslyar, D. Ge, C. Kozyrakis, and K. Olukotun, "Atlas: A chip-multiprocessor with transactional memory support," in Design, Automation Test in Europe Conference Exhibition, 2007. DATE '07, april 2007, pp. 1 -6.

[9] L. Hammond, V. Wong, M. Chen, B. D. Carlstrom, J. D. Davis, B. Hertzberg, M. K. Prabhu, H. Wijaya, C. Kozyrakis, and K. Olukotun, "Transactional memory coherence and consistency," in Proceedings of the 31st Annual International Symposium on Computer Architecture. IEEE Computer Society, Jun 2004, p. 102.

[10] C. Kachris and C. Kulkarni, "Configurable transactional memory," in Proceedings of the 15th Annual IEEE Symposium on FieldProgrammable Custom Computing Machines. Washington, DC, USA IEEE Computer Society, 2007, pp. 65-72. [Online]. Available: http://dl.acm.org/citation.cfm?id=1302498.1303053

[11] B. Saha, A.-R. Adl-Tabatabai, and Q. Jacobson, "Architectural support for software transactional memory," in MICRO 39: Proceedings of the 39th Annual IEEE/ACM International Symposium on Microarchitecture. Washington, DC, USA: IEEE Computer Society, 2006, pp. 185-196.

[12] Y. Lev, M. Moir, and D. Nussbaum, "PhTM: Phased transactional memory," in TRANSACT '07: 2nd Workshop on Transactional Computing, aug 2007.

[13] P. Damron, A. Fedorova, Y. Lev, V. Luchangco, M. Moir, and D. Nussbaum, "Hybrid transactional memory," in Proceedings of the 12th international conference on Architectural support for programming languages and operating systems, ser. ASPLOS-XII. New York, NY, USA: ACM, 2006, pp. 336-346. [Online]. Available: http://doi.acm.org/10.1145/1168857.1168900

[14] D. Christie, J.-W. Chung, S. Diestelhorst, M. Hohmuth, M. Pohlack, C. Fetzer, M. Nowack, T. Riegel, P. Felber, P. Marlier, and E. Rivière, "Evaluation of amd's advanced synchronization facility within a complete transactional memory stack," in Proceedings of the 5th European conference on Computer systems, ser. EuroSys '10. New York, NY, USA: ACM, 2010, pp. 27-40. [Online]. Available: http://doi.acm.org/10.1145/1755913.1755918

[15] D. Dice, Y. Lev, M. Moir, and D. Nussbaum, "Early experience with a commercial hardware transactional memory implementation," SIGPLAN Not., vol. 44, pp. 157-168, Mar. 2009. [Online]. Available: http://doi.acm.org/10.1145/1508284.1508263

[16] S. Chaudhry, R. Cypher, M. Ekman, M. Karlsson, A. Landin, S. Yip, H. Zeffer, and M. Tremblay, "Rock: A high-performance sparc cmt processor," Micro, IEEE, vol. 29, no. 2, pp. 6-16, march-april 2009.

[17] S. Kang and D. A. Bader, "An efficient transactional memory algorithm for computing minimum spanning forest of sparse graphs," in
PPoPP '09: Proc. 14th ACM SIGPLAN symposium on Principles and practice of parallel programming, feb 2009, pp. 15-24.

[18] C. Click, "Azul's experiences with hardware transactional memory," Jan 2009, in HP Labs - Bay Area Workshop on Transactional Memory.

[19] C. Cao Minh, J. Chung, C. Kozyrakis, and K. Olukotun, "STAMP: Stanford transactional applications for multi-processing," in IISWC '08: Proceedings of The IEEE International Symposium on Workload Characterization, September 2008.

[20] R. Haring, "The Blue Gene/Q Compute Chip," in Hot Chips 23, August 2011. [Online]. Available: url\{http://www.hotchips.org/archives/hc23/HC23-papers/HC23.18. 1-manycore/HC23.18.121.BlueGene-IBM_BQC_HC23_20110818.pdf \}

[21] G. Bronevetsky, J. Gyllenhaal, and B. R. De Supinski, "Clomp: accurately characterizing openmp application overheads," in Proceedings of the 4th international conference on OpenMP in a new era of parallelism, ser. IWOMP'08. Berlin, Heidelberg: Springer-Verlag, 2008, pp. 13-25. [Online]. Available: http://dl.acm.org/citation.cfm?id= 1789826.1789829

[22] F. Zyulkyarov, A. Cristal, S. Cvijic, E. Ayguade, M. Valero, O. Unsal, and T. Harris, "Wormbench: a configurable workload for evaluating transactional memory systems," in Proceedings of the 9th workshop on MEmory performance: DEaling with Applications, systems and architecture, ser. MEDEA '08. New York, NY, USA: ACM, 2008, pp. 61-68. [Online]. Available: http://doi.acm.org/10.1145/1509084. 1509093

[23] S. Hong, T. Oguntebi, J. Casper, N. Bronson, C. Kozyrakis, and K. Olukotun, "Eigenbench: A simple exploration tool for orthogonal tm characteristics," in Proceedings of the IEEE International Symposium on Workload Characterization (IISWC'10), ser. IISWC '10. Washington, DC, USA: IEEE Computer Society, 2010, pp. 1-11. [Online]. Available: http://dx.doi.org/10.1109/IISWC.2010.5648812

[24] B. L. Bihari, "Applicability of transactional memory to modern codes," in International Conference on Numerical Analysis and Applied Mathematics 2010 (ICNAAM 2010) Conference Proceedings. Rodos, Greece: APS, 2010, pp. 1764-1767.

[25] M. Wong, B. L. Bihari, B. R. de Supinski, P. Wu, M. M. amd Y. Liu, and W. Chen, "A case for including transactions in OpenMP," in IWOMP 2010 Conference Proceedings. Tsukuba, Japan: LNCS 6132, June 2010, pp. $149-160$.

[26] C. Bienia, "Benchmarking modern multiprocessors," Ph.D. dissertation, Princeton University, January 2011. 